\title{
Pre-discharge factors associated with unplanned readmission of patients with home mechanical ventilation: a retrospective study
}

Shwu-Jen Lin

National Taiwan University Hospital

Yao-Wen Kuo

National Taiwan University Hospital

Huey-Dong Wu

National Taiwan University Hospital

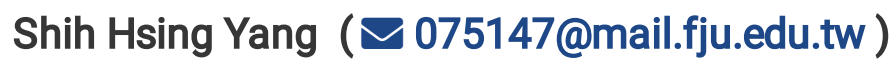

Fu Jen Catholic University

\section{Research Article}

Keywords: Unplanned readmission, pre-discharge factor, previous hospitalization, home-ventilated patient, chronic cardiovascular disease

Posted Date: March 9th, 2022

DOI: https://doi.org/10.21203/rs.3.rs-1282677/v2

License: (9) This work is licensed under a Creative Commons Attribution 4.0 International License. Read Full License 


\section{Abstract}

Exploring the characteristics and prognosis of unplanned readmissions of patients using a home ventilator and analyzing relevant pre-discharge factors that affect such unplanned readmissions.

A retrospective study was conducted to collect medical record data for 2013-2017 on the readmission of home-ventilated patients in a medical center in northern Taiwan. The average intervals of home care for the early readmission group ( $\leq 30$ days) and the late readmission group ( $\geq 31$ days) were $15.1 \pm 9.2$ and $164.8 \pm 143.2$ days, respectively. Regarding risk factors of early and late unplanned hospital readmission, the odds ratio $(\mathrm{OR})$ for patients with chronic cardiovascular disease compared with those without this disease was 4.535 (95\% Cl 1.253 -16.413). For maximum inspiratory pressure $\leqq-30 \mathrm{cmH} 20$ compared with $>-30 \mathrm{cmH} 2 \mathrm{O}$, the OR for early readmission was 0.207 (95\% $\mathrm{Cl} 0.056-0.767)$. For hemoglobin $\geq 10.1 \mathrm{~g} / \mathrm{dL}$ compared with $<10.1 \mathrm{~g} / \mathrm{dL}$, the OR of early readmission was 0.280 (95\% $\mathrm{Cl} 0.082-0.958$ ). So, pre-discharge problems, including chronic cardiovascular disease, maximum inspiratory pressure, and reduced hemoglobin, are risk factors for unplanned early hospitalization readmission of patients using a ventilator at home. Therefore, attention should be paid to these risk factors during discharge planning.

\section{Introduction}

Prolonged mechanical ventilation (PMV) has been defined as patients using a ventilator for more than 21 consecutive days for over 6 hours per day. Patients with mechanical ventilation in intensive care units need PMV-the incidence is about 6.3-14.3\% ${ }^{1-4}$. However, Scheinhorn et al found through their multicenter study regarding the discharge disposition of PMV patients in the United States that $28.8 \%$ returned home after they were discharged from hospital ${ }^{5}$. In Sweden, the prevalence rate of PMV patients increased from $6.2 / 100,000$ population in 1996 to $10.5 / 100,000$ population in 2002. Further, Lloyd-Owen studied the prevalence rate of patients using mechanical ventilators at home during 2001-2002 in 16 European countries and found that the prevalence rate of home mechanical ventilation (HMV) patients is $6.6 \%$ per 100,000 population ${ }^{6}$. Rose and colleagues investigated the prevalence of PMV patients at home in Canada and estimated that the prevalence rate is 12.9 per 100,000 population ${ }^{7}$. Therefore, PMV patients receiving home care have increased recently, but the mortality seems to be high. A 12-year population-based retrospective cohort study found that $23 \%$ of 4,670 patients needed HMV from acute care hospitalization, but their mortality rate remained unacceptably high, ranging from 8.3-49.8\% according to age group and duration of home care ${ }^{8}$. Another study has also reported the prevalence of a high mortality rate (67\%) in HMV patients aged above 65 years after they were discharged from hospital. ${ }^{2}$ Thus, it is crucial to recognize whether HMV patients face a high risk of death after discharging them from hospital because the factors related to unplanned hospital readmission also increase their mortality risk.

Unplanned hospital readmission is a concerning issue 9,10 , which has been defined as an unscheduled readmission after a patient has undergone a series of hospitalizations and been transferred to home care 
11,12 . In a systematic review, Prescott et al analyzed the early ( $\leqq 30$ days) and the late ( $\geqq 31$ days) readmission rates of pneumonia patients ${ }^{13}$. They revealed that some studies reported early readmission rates ranging from $16.8-20.1 \%$ and late readmission rates ranging from $22-35.6 \%$ after discharge up to 6 months, $46 \%$ at 12 months, and $72 \%$ after discharge up to readmission at a median 3.8 years. However, Page et al showed that the rate of hospital readmission after a 30 -day interval was $22.7 \%$ among mechanically ventilated emergency department patients ${ }^{14}$. However, although hospital readmission is a serious concern, the literature is yet to determine the relative risk factors of HMV patients that result in unplanned hospital readmissions.

In this regard, Ambrosino and colleagues presented a systematic review and meta-analysis study on the influence factors of PMV patients' ventilator dependance ${ }^{15}$. They showed that the factors related to longterm ventilator dependence include the following: (1) systemic factors: chronic diseases, comorbidities, nutrition problems, illness severity, and sepsis; (2) cardiovascular function; (3) severe neuromuscular disease; (4) respiratory factors: unresolved causes of respiratory failure, diaphragm weakness or dysfunction, imbalance between respiratory work and respiratory muscles, tracheobronchial obstruction, ineffective cough, and excessive secretions; (5) the complexity of medical quality control: ventilatorrelated pneumonia, infection, sedation, and lack of early rehabilitation; (6) cognitive factors: insomnia, anxiety, and depression; and (7) medical management factors: staffing and training problems. Further, Dettmer et al highlighted that the ventilator dependence of PMV patients is influenced by mortality-related factors ${ }^{16}$, such as age, use of blood pressure drugs, thrombocytopenia, pre-existing kidney disease, respiratory failure and acute kidney injury, and the need for hemodialysis.

In a prospective study of 297 patients in Australia aged above 60 years, Sharma et al. found that the nutritional status of elderly patients at admission can predict early or late unplanned hospital readmission and death after discharge ${ }^{17}$. The average age of participants in their study was 80.3 years, 64.3\% were female, the patients had multiple comorbidities, and the average Charlson Comorbidity Index was 2.3 points. In the diagnosis of diseases, respiratory diseases, sepsis, and cardiovascular diseases were the three main diagnoses. However, it is unclear what the impact factors cause unplanned hospital readmissions of HMV patients, and whether the factors are relative states of patients in the pre-discharge period of the previous hospitalization.

Therefore, the present study explored the following questions: (1) What was the status of unplanned hospital readmission of HMV patients? (2) According to data collected for different time intervals of unplanned hospital readmission from pre-discharge during previous hospitalization, what were the demographic characteristics, the disease attributes, the physiological parameters, the respiratory parameters of these patients? (3) What were the main relevant factors during the previous hospitalization of HMV patients who experienced unplanned hospital readmissions?

Thus, the purpose of this study was to explore the pre-discharge factors regarding hospitalization associated with unplanned hospital readmission of patients using HMV. To this end, we conducted a 
retrospective analysis of data on HMV patients who experienced unplanned hospital readmission, including their demographic characteristics, hospitalization data, and respiratory parameters.

\section{Results}

\section{Unplanned hospital readmission in HMV patients}

Table 1 presents the population data. Although the results did not reveal any significant difference according to year, the readmission rate (47.2\%) for the interval of 31-180 days after discharge was more than that for the others, although the rate for the interval of $\leq 30$ days was also high (33.1\%). However, this rate decreased sharply for the intervals of 181-365 and $\geq 366$ days. Thus, PMV patients experienced unplanned hospital readmission after the previous discharge, frequently within less than 180 days after being discharged to their homes and undergoing home care, and many of them were readmitted even less than 30 days after discharge, which is a highly significant finding.

Table 1

The rate of unplanned hospital readmission by several interval days in-home mechanical ventilator patients

\begin{tabular}{|c|c|c|c|c|c|c|}
\hline \multirow[t]{2}{*}{ Year } & \multirow[t]{2}{*}{ Number } & \multicolumn{4}{|c|}{ Interval day of unplanned hospital readmission } & \multirow[t]{2}{*}{ P-value } \\
\hline & & $\leq 30$ days & $31-180$ days & $181-365$ days & $\geq 366$ days & \\
\hline 2013 & 14 & $3(21.4 \%)$ & $8(57.1 \%)$ & $1(7.1 \%)$ & $2(14.3 \%)$ & \multirow[t]{6}{*}{0.338} \\
\hline 2014 & 27 & $7(25.9 \%)$ & $17(63.0 \%)$ & $3(11.1 \%)$ & $0(0 \%)$ & \\
\hline 2015 & 37 & $12(32.4 \%)$ & $14(37.8 \%)$ & $7(18.9 \%)$ & $4(10.8 \%)$ & \\
\hline 2016 & 26 & $11(42.3 \%)$ & $9(34.6 \%)$ & $5(19.2 \%)$ & $1(3.8 \%)$ & \\
\hline 2017 & 23 & $9(39.1 \%)$ & $12(52.2 \%)$ & $1(4.3 \%)$ & $1(4.3 \%)$ & \\
\hline Total & 127 & $42(33.1 \%)$ & $60(47.2 \%)$ & $17(13.4 \%)$ & $8(6.3 \%)$ & \\
\hline
\end{tabular}

\section{Factors associated with demographic and characteristics}

HMV patients were further grouped into the early and late unplanned hospital readmission groups. The cut-off number was 30 days, with those readmitted before 31 days after the previous discharge categorized into the former group and the others into the latter group. Analysis factors regarding demographic changes are shown in Table 2. The number of female patients was more than that of male patients. The mean age of the two groups was similar $(69.9 \pm 21.3$ for the early readmission group vs $68.9 \pm 19.2$ for the late readmission group). The weight and the Body Mass Index (BMI) of the two groups differed significantly, but both groups had very few patients with BMI > 30. Next, the ventilator-dependent days during the previous admission of the early readmission group were two times more than that of the late readmission group ( $81.4 \pm 78.8$ vs $47.5 \pm 36.6$ ), and the duration of hospital stay on previous admission of the former group $(88.1 \pm 77.5)$ was also more than that of the latter group $(51.9 \pm 39.9)$. In 
addition, the average interval of home care for the early readmission group ( $15.1 \pm 9.2$ days) was significantly shorter than that for the late readmission group (164.8 \pm 143.2 days).

Table 2

Demographic changes of home ventilated patients with unplanned hospital readmission

\begin{tabular}{|c|c|c|c|}
\hline \multirow{2}{*}{ Variables } & \multicolumn{2}{|c|}{ Interval day of unplanned hospital readmission } & \multirow[t]{2}{*}{ P-value } \\
\hline & $\leq 30$ days & $\geq 31$ days & \\
\hline Number & 42 & 85 & \\
\hline \multicolumn{4}{|l|}{ Gender $^{a}$} \\
\hline Man & $16(38.1 \%)$ & $43(50.6 \%)$ & 0.184 \\
\hline Female & $26(61.9 \%)$ & $42(49.4 \%)$ & \\
\hline Age $^{b}$ & $69.9 \pm 21.3(20.3 \sim 96.3)$ & $68.9 \pm 19.2(20.0 \sim 92.6)$ & 0.797 \\
\hline Height $(\mathrm{cm})^{b}$ & $158.3 \pm 8.6(140.0 \sim 175.0)$ & $159.7 \pm 9.3(139.0 \sim 78.0)$ & 0.417 \\
\hline Weight $(\mathrm{kgw})^{\mathrm{b}}$ & $52.0 \pm 16.2(29.0 \sim 121.0)$ & $59.4 \pm 17.7(26.0 \sim 118.0)$ & $0.024^{\star}$ \\
\hline BMI $\left(\mathrm{kg} / \mathrm{m}^{2}\right)^{\mathrm{b}}$ & $20.8 \pm 5.8(10.0 \sim 40.9)$ & $23.3 \pm 6.4(10.8 \sim 46.2)$ & $0.036^{\star}$ \\
\hline \multicolumn{4}{|l|}{ Obesity ${ }^{a}$} \\
\hline Normal & $20(48.2 \%)$ & $41(48.0 \%)$ & 0.051 \\
\hline Underweight & $13(12.9 \%)$ & $11(18.9 \%)$ & \\
\hline Overweight & $5(16.5 \%)$ & $14(15.0 \%)$ & \\
\hline Obese & $4(9.5 \%)$ & $19(22.4 \%)$ & \\
\hline \multicolumn{4}{|l|}{ Education level a } \\
\hline Illiterate or elementary school & $16(38.1 \%)$ & $33(38.8 \%)$ & 0.929 \\
\hline Junior high school & $3(7.1 \%)$ & $9(10.6 \%)$ & \\
\hline Senior high school & $7(16.7 \%)$ & $13(15.3 \%)$ & \\
\hline College or above & $16(38.1 \%)$ & $30(35.3 \%)$ & \\
\hline Ventilator days ${ }^{b}$ & $81.4 \pm 78.8(2 \sim 432)$ & $47.5 \pm 36.6(6 \sim 163)$ & $0.001^{\star}$ \\
\hline Admission days ${ }^{\mathrm{b}}$ & $88.1 \pm 77.5(2 \sim 432)$ & $51.9 \pm 39.9(2 \sim 178)$ & $0.001^{*}$ \\
\hline Interval days after discharge ${ }^{b}$ & $15.1 \pm 9.2(2 \sim 30)$ & $164.8 \pm 143.2(31 \sim 813)$ & $<0.001^{\star}$ \\
\hline
\end{tabular}




\section{Analysis of clinical factors}

A few patients had a history of smoking and alcohol consumption but there were no significant differences between the two groups in this regard, as shown in Fig. 2. However, regarding the history of chronic disease, the HMV patients had hypertension and cardiovascular disease, and the number of cases in the early readmission group was significantly more than that in the late readmission group. Further, the Charlson Comorbidity Index of the early readmission group $(2.9 \pm 2.2)$ was significantly higher than that of the late readmission group $(1.7 \pm 1.5)$. Next, Fig. 3 shows that the factors regarding therapeutic tubing of patients was not significantly different for these two groups. An analysis of daily activity and assistance from caregivers showed that the percentage of foreign nursing workers was the highest. Our analysis of the diagnosis on admission in the previous hospitalization (Fig. 2e) revealed that the significant factors of early unplanned hospital readmission were lung disease and infection-related diseases. Further, we compared factors regarding respiratory parameters (Fig. 4) and hemodynamic and laboratory data (Fig. 5a, 5b). The early readmission group's rate of use of oxygen from the home ventilator (3.4 \pm 1.2$)$ was significantly higher than the rate of the late readmission group $(2.5 \pm 1.3)$. Maximal inspiratory pressure (PImax), maximal expiratory pressure (PEmax), and rapid shallow breathing index (RSBI) of the weaning profile were better for the late readmission group compared with the early readmission group. The factor of hemodynamic data was not significant. The laboratory data, such as the white blood cell count and the hemoglobin, potassium, and magnesium levels, of the patients in the late readmission group were significantly better than the data of those in the early readmission group.

\section{Multivariate logistic regression analysis for the significant risk factors}

We used the significant risk factors for early rather than late unplanned hospital readmission for HMV patients in a multivariate logistic regression analysis (see Table 3). The significant odds ratio indicated that PImax, the hemoglobin level, and a history of cardiovascular disease, were the main risk factors that can lead to readmission less than 30 days after discharge. Having cardiovascular problems in HMV patients even presents a high to fourfold risk of early readmission after discharge.

Table 3

Multivariate logistic regression analysis for the significant risk factors

\begin{tabular}{|lcccccc|}
\hline Variables & B & SE & OR & $95 \%$ Cl & P-value \\
\hline PImax & & & & Lower limit & Upper limit & \\
\hline Hemoglobin & -1.575 & 0.668 & 0.207 & 0.056 & 0.767 & $0.018^{*}$ \\
\hline Cardiovascular diseases & 1.512 & 0.656 & 4.535 & 1.253 & 16.413 & $0.021^{*}$ \\
\hline $\begin{array}{l}\text { In the multivariate logistic regression model, Abbreviations: B: regression coefficient, SE: standard } \\
\text { error, OR: odds ratio. }\end{array}$ & -1.272 & 0.627 & 0.280 & 0.082 & 0.958 & $0.043^{*}$ \\
\hline
\end{tabular}




\section{Discussion}

In this study, we revealed risk factors for preventing unplanned hospital readmission after discharge of PMV patients receiving home care. This study mainly aimed to explore the relative risk factors in the predischarge period during previous hospitalization to prevent the unplanned hospital readmission of these patients. PImax, the hemoglobin level, and a history of cardiovascular disease were the main risk factors that can lead to readmission less than 30 days after discharge. The results are significant because these imply that HMV patients' chances of avoiding readmission after discharge depend on their recovery levels, mainly their respiratory muscle strength and oxygen delivery ability, during the pre-discharge period. Clinical care can use the findings of this study for reference.

The prevalence of unplanned hospital readmissions among HMV patients has attracted limited research attention. Although the present study is not a prospective or a cohort-design study, it comprehensively expressed the percentage of unplanned readmissions every year in the subsequent five years. A significant finding of this study for improving the quality of clinical care is that the percentage of early readmission in the $\leq 30$ days interval after discharge was very high; it was the highest in the 31-180 days interval, and even in the 30 days group, the average interval was 15 days. Thus, this finding clarifies the timing of readmission risk among HMV patients receiving home care. Lindenauer et al analyzed data on 226,545 patients in 4,675 hospitals in the United States, who were aged more than 65 years and were hospitalized owing to a diagnosis of pneumonia ${ }^{18}$. The 30 -day readmission rate was $17.4 \%$, and among these patients, $55.5 \%$ were females. In addition, Douglas et al conducted a larger prospective longitudinal descriptive study to identify the risk factors for readmission for 199 patients in the intensive care unit with a history of long-term use of ventilators ${ }^{19}$. They found that the readmission rate was $21.1 \%$ at 30 days after hospital discharge, which increased to $34.2 \%$ at 60 days after discharge. For the 1 to 60 days interval, the average number of days of hospitalization on readmission was 39.2 days and the median was 19 days; however, the hospital readmission rate for the 180-day interval was $8 \%$. In addition, $38.2 \%$ of the patients were readmitted at least once, and $14.6 \%$ had been hospitalized 2-7 times. Therefore, the results of the present study are similar to those of Douglas's study, which showed a very high readmission during the 1 to 60 days after discharge among patients with a long-term use of ventilators.

Further, we explored the risk factors for the unplanned hospital readmission of HMV patients. Our study explored the readmission factors that focused on pre-discharge about hospitalization parameters of recovery status during previous hospitalization in HMV patients. Graham et al have analyzed numerous types of data on patients' admission in the United States ${ }^{20}$. Their prospective cohort study analyzed data from 10 academic medical centers with a total of 822 general ward patients in order to determine the risk factors of readmission after discharge for preventing readmission. Their results showed risk factors; there were $301(36.6 \%)$ early readmissions and $521(63.4 \%)$ late readmissions. The mean ages for the early and late readmission groups were 54.7 and 55.5 years, respectively. As regards these patients' history of chronic diseases, patients with heart failure function grade III or IV accounted for $15.0 \%$ and $12.9 \%$ of the total readmissions. The authors also pointed out that $36.2 \%$ of early readmissions and $23 \%$ of late readmissions can be prevented. The risk factor of history of cardiovascular disease was significantly 
manifested in patients who were readmitted in an earlier time interval, which is a finding similar to that of the present study. In addition, a prospective study of 297 patients older than 60 years by Sharma and other scholars explored whether the nutritional status of elderly patients at admission predicts early or late unplanned readmission or death after discharge ${ }^{17}$. The average age was 80.3 years, $64.3 \%$ of these patients were females, and the average number of comorbidities was 6.2. The average Charlson Comorbidity Index was 2.3 points. In the diagnosis of diseases, respiratory diseases, sepsis, and cardiovascular diseases were the three main diagnoses. It is specifically demonstrated that the malnutrition of elderly patients upon admission is an important predictor of early readmission or death after hospital discharge. However, the exploratory strategy we adopted in this study allowed us to use hospitalization data as a risk factor, and to consider whether the risk factor of readmission is associated with hospitalization parameters at pre-discharge. Our study differs from other studies in that we focused on HMV patients.

To identify the risk factors of unplanned hospital readmission in the present study, we analyzed the previous hospitalization data about demographics, laboratory data, and clinical and respiratory parameters at pre-discharge in HMV patients. A comparison of early and late readmission showed that significant risk factors were patients' weight, BMI, history of hypertension and cardiovascular disease, the comorbidity index for history of disease, the lack of a caregiver, and a diagnosis of pulmonary and infectious diseases. The risk factors associated with mechanical ventilation were the rate of oxygen use, PImax, PEmax, and RSBI for the weaning profiles. Analysis of clinical laboratory data showed that the significant risk factors were the white blood count, and the levels of hemoglobin, potassium, and magnesium. The significant risk factors were worse for the early readmission group than for the late readmission group. In a survey in the United States by Scheinhorn et al, the average age of PMV patients was 71.4 years ${ }^{5}$. The average age of PMV patients in Italy, as Polverino's study reported, was more than 70 years ${ }^{21}$. In the meta-analysis study of Rodakowski and colleagues ${ }^{22}$, the demographic characteristics of caregivers showed that their age varied greatly; further, $34 \%$ of caregivers were men, of which $61 \%$ are spouses or partners and $35 \%$ are their adult children. In the elderly patient discharge plan, the impact of using the integrated discharge plan was compared with the normal discharge plan. After the implementation of the integrated discharge plan, the elderly patients' 90 -day readmission reduced by $25 \%$, and 180 days after the implementation of the integrated discharge plan, hospital admissions decreased by $24 \%$. A study by British scholars, such as Lone et al, also showed that pneumonia, chronic obstructive pulmonary disease, sepsis, and decompensated congestive heart failure were the most common causes of respiratory failure in patients requiring PMV ${ }^{4}$. Prescott et al conducted a systematic review of studies from the United States, Spain, Canada, Croatia, and Sweden on the readmission of patients with pneumonia after discharge ${ }^{13}$. The most common readmission diagnoses are pneumonia, heart failure and cardiovascular disease, chronic obstructive pulmonary disease and lung disease, and sepsis, despite subjects without HMV, which is similar to the results of our study. The present study showed that for hemoglobin $\geq 10.1 \mathrm{~g} / \mathrm{dL}$ compared with $<10.1 \mathrm{~g} / \mathrm{dL}$, the risk ratio of readmission within 30 days significantly reduced by 0.280 times. Similarly, it is predicted that the increased unplanned readmissions within 30 days for every unit of hemoglobin reduction among general medical inpatients 
will increase the readmission rate by nearly $4 \%{ }^{23}$. In addition, the present study found that the risk of readmission within 30 days on setting the oxygen flow rate of the home ventilator at $\geq 3 \mathrm{~L} / \mathrm{min}$ was 4.151 times higher than the risk on setting this rate at $<3 \mathrm{~L} / \mathrm{min}$. A comparison of PImax $<-30 \mathrm{cmH} 2 \mathrm{O}$ and $\geq-$ $30 \mathrm{cmH} 20$ showed that the risk ratio of readmission within 30 days was reduced to 0.207 times in the latter case. Supinski et al analyzed data on adult patients in the Intensive Care Unit of the University of Kentucky Department of Internal Medicine who used ventilators. They found that the patient's PImax measurements were statistically significant and affected the patients' ventilator use time and survival ${ }^{24}$. Hamazaki et al studied 456 patients in Japan with congestive heart failure who received cardiac rehabilitation for 5 months during hospitalization and after discharge ${ }^{25}$. They revealed that the higher the PImax was, the higher was the survival rate and that it was even an independent predictor of patient readmission and mortality. Mikkelsen et al reported that improvement in exercise capacity during a cardiac rehabilitation program was highly predictive of the future risk of readmissions for cardiovascular disease and mortality ${ }^{26}$. Thus, the results of these studies are all similar to our results, which indicate that hemoglobin, circulation, and respiratory muscle strength affect oxygen delivery to cover oxygen consumption during exercise capacity, and thus, help patients to avoid readmission. Therefore, if patients can cope with the daily activity at home, which is dependent on the level of cardiopulmonary function recovery and endurance at pre-discharge, they can avoid hospital readmission. Conversely, the consequence of inefficient coping by HMV patients may be unplanned hospital readmissions.

\section{Methods}

\section{Study design}

The retrospective study was conducted in a medical center in Taipei, Taiwan. The patients' hospitalization data from January 1, 2013 to December 31, 2017 were collected from the hospital's REDCap (Research Electronic Data Capture, REDCap) system, which is a clinical research electronic data capture system.

\section{Ethics approval and consent to participate.}

This study was reviewed and approved by the Institutional Review Board and Ethics Committee, which is Research Ethics Committee D (R.E.C.D) of the National Taiwan University Hospital (NTUH), Taipei City, Taiwan, and all methods were performed in accordance with the relevant guidelines and regulations. The R.E.C.D has also waived the need for informed consent as retrospective study (approval number, NTUHREC No. 201901054RIND).

\section{Selection criteria.}

The inclusion criteria used to select subjects for this study were as follows: (1) The selected patients were ventilator-dependent and received home care from National Taiwan University Hospital (NTUH). Ventilator dependence has been defined by the Integrated Delivery System (IDS) in Taiwan ${ }^{3,27}$. (2) The patents were 
adults aged more than 20 years. (Taiwan's legal age of majority is 20 years, and the IDS project included 20-year-old patients.) Patients were excluded if (1) they had not been readmitted to NTUH after discharge; (2) their readmission was planned; and (3) they had experienced repeated hospital readmissions. Figure 1 illustrates this selection process. From 2013 to 2017, the number of IDS cases in NTUH was 6,925 of which 913 were HMV patients. After excluding patients aged less than 20 years, those who were not readmitted, 16 planned readmissions, and 295 repeated readmissions, the final study sample consisted of 127 patients, early readmission group had 42 patients, late readmission group of 85 . We confirmed that the sample size was adequate by using $G$ Power (version 3.1.9.2) statistical software. Estimates showed that the verification power increased to $90 \%$ (power $=0.9$ ) for a sample size of 117 . Thus, the sample size of this study, which exceeds 117 , can be considered satisfactory.

\section{Data management and statistical analysis.}

The collected parameters for the subjects included their basic characteristics before discharge during the previous hospitalization. We included the hospitalization data relevant to this study, such as the recorded clinical data from the laboratory, the respiratory parameters of ventilator status, and weaning profiles. We imported the data into SPSS version 18.0 statistical software for statistical analysis.

Descriptive statistics describe the distribution of demographic variables included in this study ${ }^{10}$. The subjects were grouped according to the number of days (ie, $\leqq 30,31-180,181-365, \geq 366$ days) between their discharge from the previous hospitalization and their readmission and were divided into an early readmission group ( $\leq 30$ days) and a late readmission group ( $\geq 31$ days). Further, we used the independent sample $t$ test by Student's $t$-test or chi-square test to test significant differences in invariances. Then, we used multiple logistic regression to analyze the influencing factors of the univariate analysis results that may be related to unplanned hospital readmissions. A $P$-value of $<0.05$ was considered a statistically significant difference for all analyses.

\section{Limitations of this study}

This study has some limitations, one of which is the research design, in that it included subjects from only one medical center; however, it used a long-term database of 5 years for retrospective analysis. In addition, the database was also available only for 5 years, that is, from 2013 to 2017 . Moreover, the study did not include a control group of patients who have not been readmitted after discharge, and the date of the collected data is close to the pre-discharge date; nevertheless, these dates vary. However, a prospective study design on this theme is expected to be conducted. We also consider adding study design of using national database like the nationwide readmission database may give more power and generalizability and should be taken up as future studies ${ }^{28}$. And machine learning derived models is also interested, that may effectively identify high-risk patients for reduce unplanned readmissions ${ }^{29}$.

\section{Conclusion}


Home ventilator patients frequently progress to unplanned hospital readmission. This study revealed the related risk factors, which were highly associated with the recovery conditions at pre-discharge during the previous hospitalization. The hemoglobin level, inspiratory muscle strength and still presenting cardiovascular problem were closely related risk factors. The results indicate that if patients lack coping ability, oxygen delivery, and endurance during home care, this situation may lead to unplanned hospital readmissions.

\section{Abbreviations}

PMV: Prolonged Mechanical Ventilation;

HMV: Home Mechanical Ventilation;

REDCap: Research Electronic Data Capture;

NTUH: National Taiwan University Hospital;

IDS: Integrated Delivery System;

BMI: Body Mass Index;

PImax: Maximal Inspiratory Pressure;

PEmax: Maximal Expiratory Pressure;

RSBI: Rapid Shallow Breathing Index.

GCS, Glasgow Coma Scale.

B: Regression Coefficient,

SE: Standard Error,

OR: Odds Ratio.

\section{Declarations}

Data availability

The datasets used and analyzed during the current study are available from the corresponding author on reasonable request.

Acknowledgments 
We thank Chin Jung Liu, Ph.D., RRT, Associate Professor, and Chia-Chen Chu, Ph.D., RRT, Assistant Professor, as our respected advisors.

Authors' contribution

Shwu-Jen Lin and Shih Hsing Yang conceived the idea, designed, carried out the study, performed the statistical analysis and were the main writers of the manuscript. Yao-Wen Kuo and Huey-Dong Wusupervised the study for data interpretation and manuscript writing. All authors read and approved the final manuscript.

\section{Authors' information}

${ }^{1}$ Department of Integrated Diagnostics \& Therapeutics, National Taiwan University Hospital, Taipei, Taiwan.; ${ }^{2}$ Master Program in Transdisciplinary Long-Term Care, Fu Jen Catholic University, New Taipei City, Taiwan.; ${ }^{3}$ Department of Respiratory Therapy, Fu Jen Catholic University, New Taipei City, Taiwan.

Competing interests

The authors report no conflicts of interest in this work.

Funding

This work has not funded.

\section{References}

1. Cox, C. E. et al. Differences in one-year health outcomes and resource utilization by definition of prolonged mechanical ventilation: a prospective cohort study. Crit Care 11, R9, doi:10.1186/cc5667 (2007).

2. Estenssoro, E. et al. Shock on admission day is the best predictor of prolonged mechanical ventilation in the ICU. Chest 127, 598-603, doi:10.1378/chest.127.2.598 (2005).

3. Hung, M. C. et al. Life expectancies and incidence rates of patients under prolonged mechanical ventilation: a population-based study during 1998 to 2007 in Taiwan. Crit Care 15, R107, doi:10.1186/cc10128 (2011).

4. Lone, N. I. \& Walsh, T. S. Prolonged mechanical ventilation in critically ill patients: epidemiology, outcomes and modelling the potential cost consequences of establishing a regional weaning unit. Crit Care 15, R102, doi:10.1186/cc10117 (2011).

5. Scheinhorn, D. J. et al. Post-ICU mechanical ventilation at 23 long-term care hospitals: a multicenter outcomes study. Chest 131, 85-93, doi:10.1378/chest.06-1081 (2007).

6. Lloyd-Owen, S. J. et al. Patterns of home mechanical ventilation use in Europe: results from the Eurovent survey. Eur Respir J 25, 1025-1031, doi:10.1183/09031936.05.00066704 (2005). 
7. Rose, L. et al. Home mechanical ventilation in Canada: a national survey. Respir Care 60, 695-704, doi:10.4187/respcare.03609 (2015).

8. Povitz, M. et al. Home Mechanical Ventilation: A 12-Year Population-Based Retrospective Cohort Study. Respir Care 63, 380-387, doi:10.4187/respcare.05689 (2018).

9. Vasireddy, D., Sehgal, M. \& Amritphale, A. Risk Factors, Trends, and Preventive Measures for 30-Day Unplanned Diabetic Ketoacidosis Readmissions in the Pediatric Population. Cureus 13, e19205, doi:10.7759/cureus.19205 (2021).

10. Sehgal, M. et al. Demographics and Risk Factors of Pediatric Pulmonary Hypertension Readmissions. Cureus 13, e18994, doi:10.7759/cureus.18994 (2021).

11. Bohannon, R. W. \& Maljanian, R. D. Hospital readmissions of elderly patients hospitalized with pneumonia. Conn Med 67, 599-603 (2003).

12. Wong, E. L. et al. Unplanned readmission rates, length of hospital stay, mortality, and medical costs of ten common medical conditions: a retrospective analysis of Hong Kong hospital data. BMC Health Serv Res 11, 149, doi:10.1186/1472-6963-11-149 (2011).

13. Prescott, H. C., Sjoding, M. W. \& Iwashyna, T. J. Diagnoses of early and late readmissions after hospitalization for pneumonia. A systematic review. Ann Am Thorac Soc 11, 1091-1100, doi:10.1513/AnnalsATS.201404-1420C (2014).

14. Page, D. B. et al. Thirty-day hospital readmissions among mechanically ventilated emergency department patients. Emerg Med J 35, 252-256, doi:10.1136/emermed-2017-206651 (2018).

15. Ambrosino, N. \& Vitacca, M. The patient needing prolonged mechanical ventilation: a narrative review. Multidiscip Respir Med 13, 6, doi:10.1186/s40248-018-0118-7 (2018).

16. Dettmer, M. R. et al. Prognostic Factors for Long-Term Mortality in Critically III Patients Treated With Prolonged Mechanical Ventilation: A Systematic Review. Crit Care Med 45, 69-74, doi:10.1097/CCM.0000000000002022 (2017).

17. Sharma, Y. et al. Malnutrition and its association with readmission and death within 7 days and 8180 days postdischarge in older patients: a prospective observational study. BMJ Open 7, e018443, doi:10.1136/bmjopen-2017-018443 (2017).

18. Lindenauer, P. K. et al. Development, validation, and results of a measure of 30-day readmission following hospitalization for pneumonia. J Hosp Med 6, 142-150, doi:10.1002/jhm.890 (2011).

19. Douglas, S. L., Daly, B. J., Brennan, P. F., Gordon, N. H. \& Uthis, P. Hospital readmission among longterm ventilator patients. Chest 120, 1278-1286, doi:10.1378/chest.120.4.1278 (2001).

20. Graham, K. L. et al. Preventability of Early Versus Late Hospital Readmissions in a National Cohort of General Medicine Patients. Ann Intern Med 168, 766-774, doi:10.7326/M17-1724 (2018).

21. Polverino, E. et al. Patients' characterization, hospital course and clinical outcomes in five Italian respiratory intensive care units. Intensive Care Med 36, 137-142, doi:10.1007/s00134-009-1658-2 (2010). 
22. Rodakowski, J. et al. Caregiver Integration During Discharge Planning for Older Adults to Reduce Resource Use: A Metaanalysis. J Am Geriatr Soc 65, 1748-1755, doi:10.1111/jgs.14873 (2017).

23. Lin, R. J., Evans, A. T., Chused, A. E. \& Unterbrink, M. E. Anemia in general medical inpatients prolongs length of stay and increases 30-day unplanned readmission rate. South Med J 106, 316-320, doi:10.1097/SMJ.0b013e318290f930 (2013).

24. Supinski, G. S., Westgate, P. \& Callahan, L. A. Correlation of maximal inspiratory pressure to transdiaphragmatic twitch pressure in intensive care unit patients. Crit Care 20, 77, doi:10.1186/s13054-016-1247-z (2016).

25. Hamazaki, N. et al. 298Change in respiratory muscle strength predicts clinical events in patients with chronic heart failure. European Heart Journal 40, doi:10.1093/eurheartj/ehz747.0095 (2019).

26. Mikkelsen, N. et al. Improvement in VO2peak predicts readmissions for cardiovascular disease and mortality in patients undergoing cardiac rehabilitation. Eur J Prev Cardio/ 27, 811-819, doi:10.1177/2047487319887835 (2020).

27. Su, J. et al. Experience with a step-down respiratory care center at a tertiary referral medical center in Taiwan. J Crit Care 21, 156-161, doi:10.1016/j.jcrc.2005.10.004 (2006).

28. Amritphale, A., Fonarow, G. C., Amritphale, N., Omar, B. \& Crook, E. D. All-Cause Unplanned Readmissions in the United States. Insights from the Nationwide Readmission Database. Intern Med J, doi:10.1111/imj.15581 (2021).

29. Amod Amritphale et al. Predictors of 30-Day Unplanned Readmission After Carotid Artery Stenting Using Artificial Intelligence. Advances in Therapy 38, 2954-2972 (2021).

30. Shah, S., Vanclay, F. \& Cooper, B. Improving the sensitivity of the Barthel Index for stroke rehabilitation. J Clin Epidemiol 42, 703-709, doi:10.1016/0895-4356(89)90065-6 (1989).

\section{Figures}




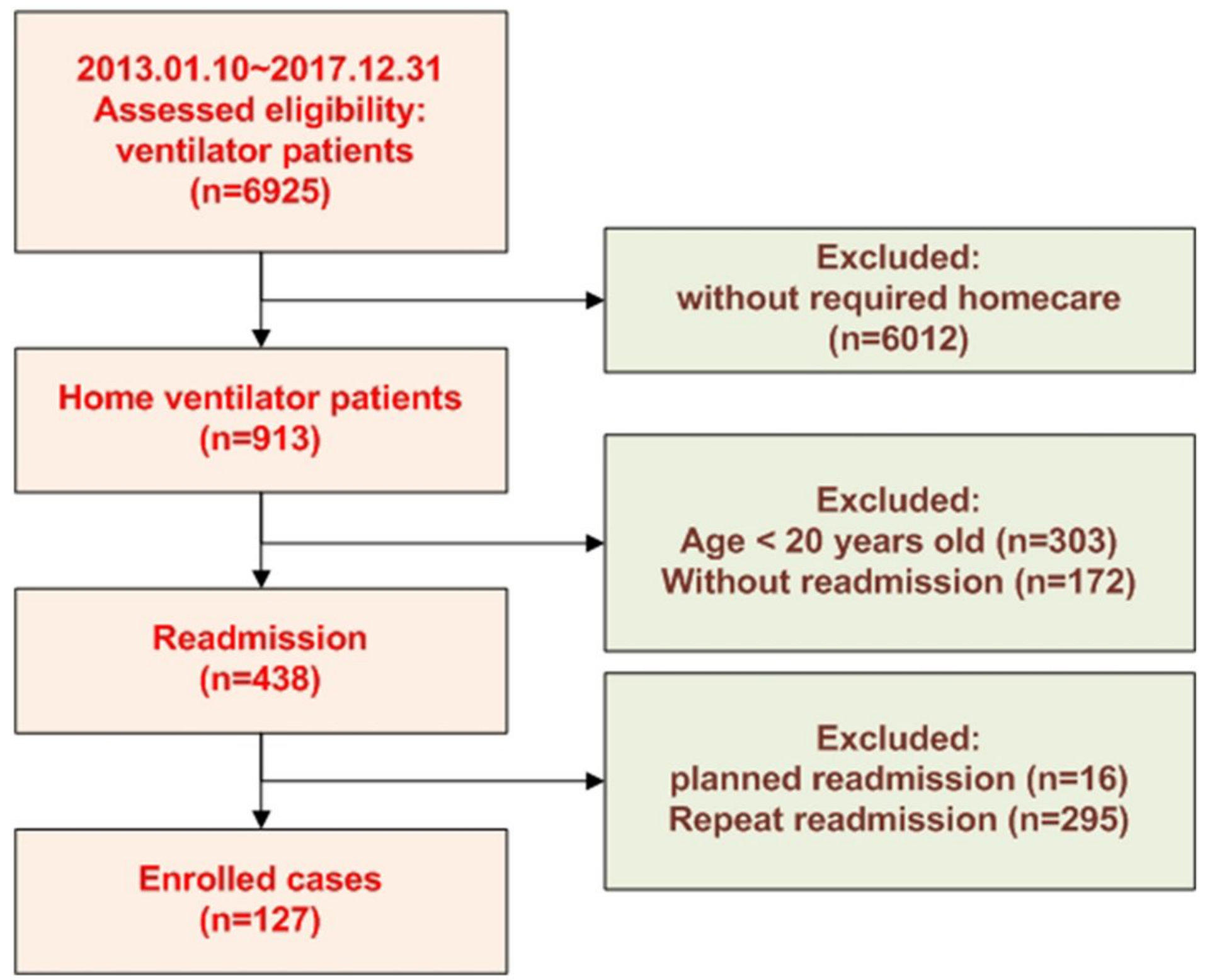

Figure 1

Flow diagram for cases analyzed. 
a

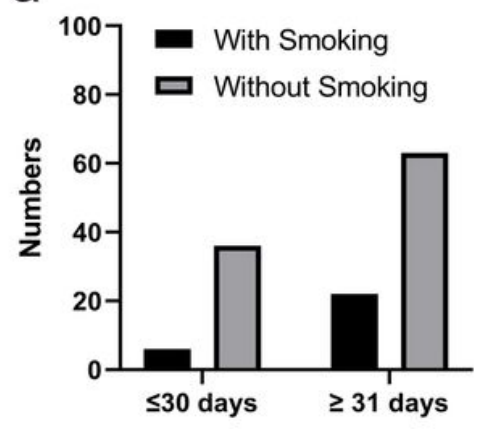

b

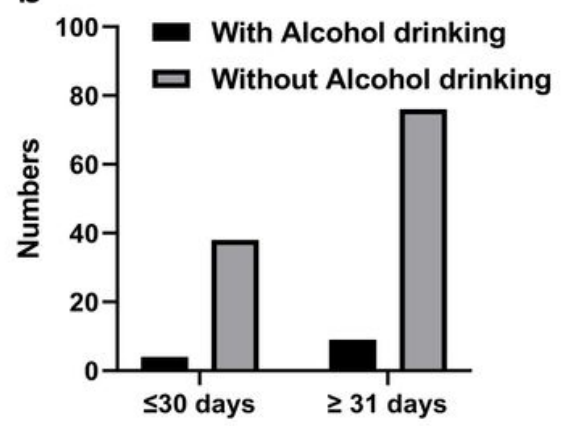

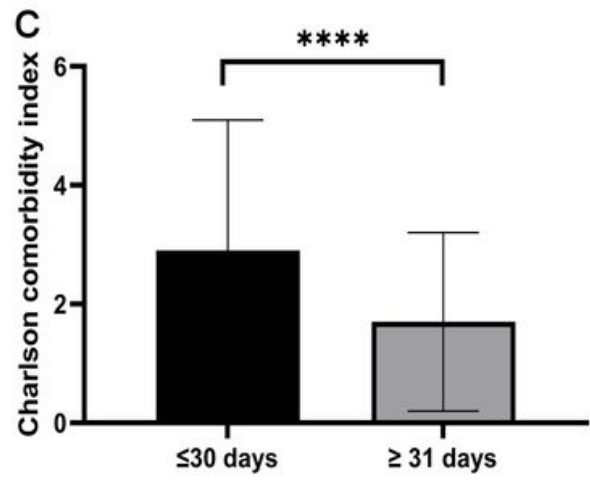

\section{d.Chronic disease history}

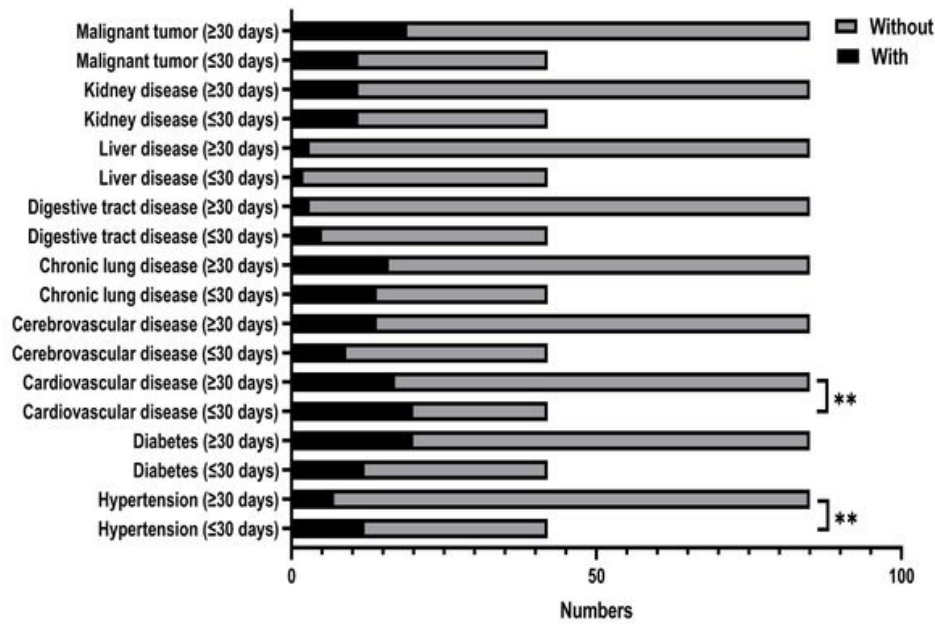

e.Medical diagnosise

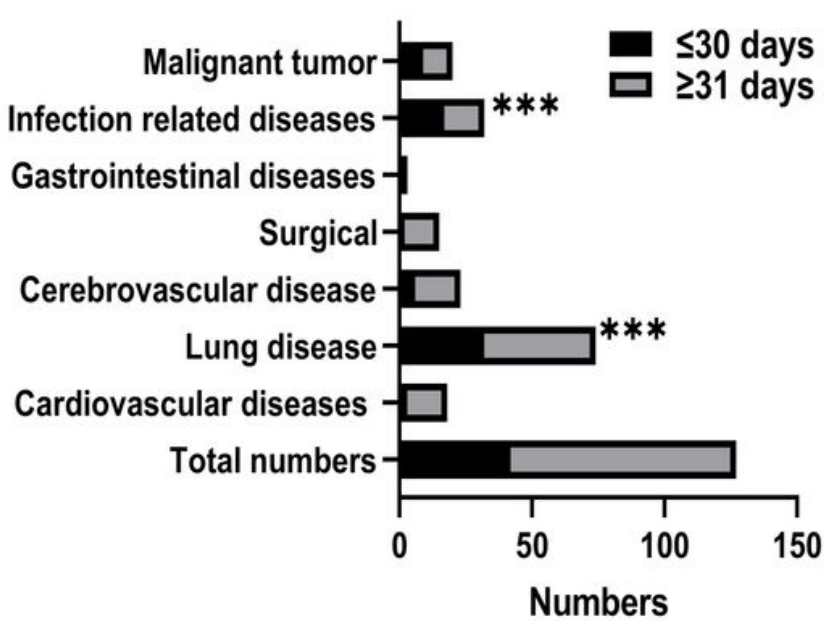

Figure 2

Risk factors for unplanned hospital readmission on history of habit and chronic disease in HMV patients.

(a), (b), (d), (e) \numbers, chi-square test; (c) 『mean $\pm S D$, independent $T$ test; ${ }^{\star \star} p<0.01,{ }^{* \star \star} p<0.001$, $\star \star \star \star p<0.0001,:$ significantly different 

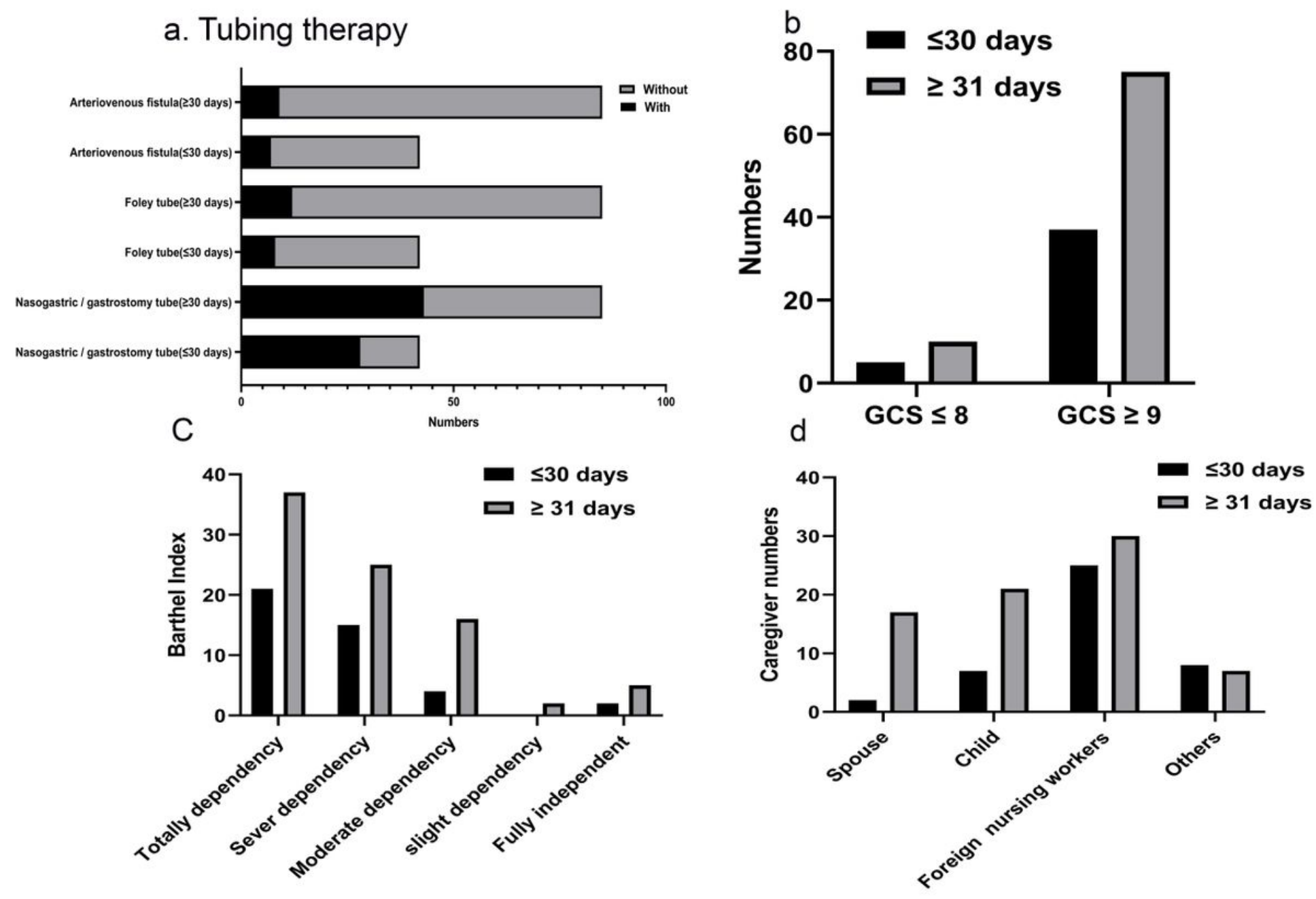

Figure 3

The factors regarding therapeutic tubing of patients, daily activity, and caregiver. Interpreting Barthel index scores. scores of 0-20 is total dependency, 21-60 is severe dependency, 61-90 indicate moderate dependency, 91-99 is slight dependency, and 100 indicate fully independent ${ }^{30}$. (d): P value $=0.029$ by chi-square test indicates a statistically significant difference. 

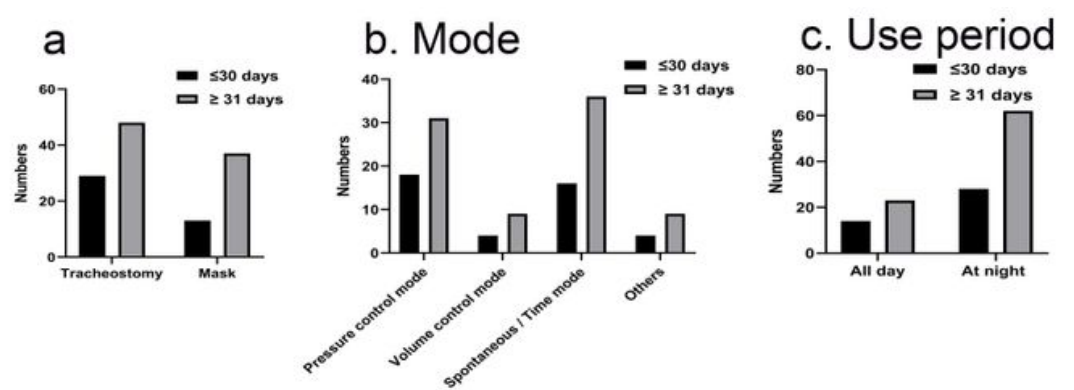

d. Ventilator setting
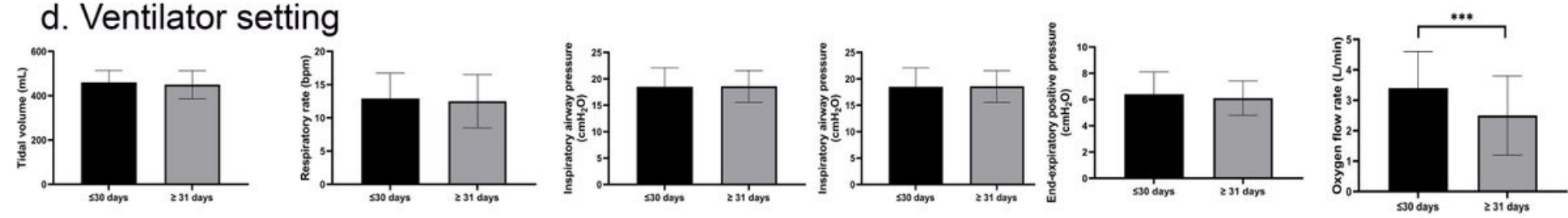

e. weaning profile
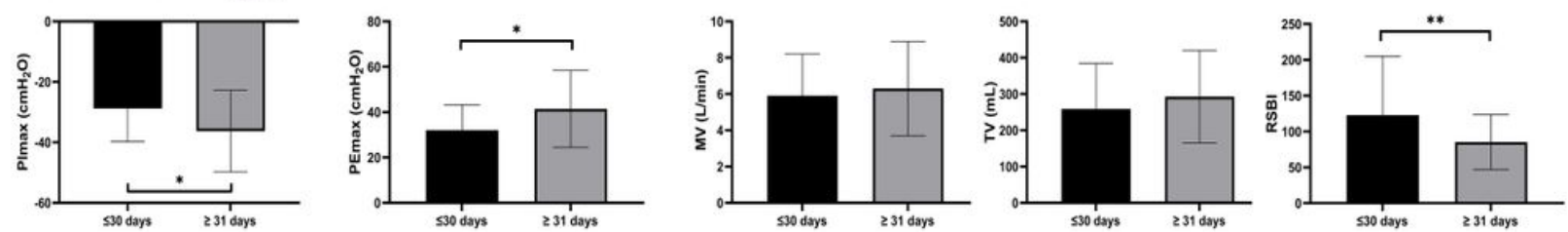

Figure 4

Comparison of early with late unplanned hospital readmission on the factors of respiratory parameters in HMV patients. (a), (b), (c) 囚numbers, chi-square test; (d), (e)囚mean $\pm S D$, independent $T$ test; ${ }^{*} p<0.05$, $\star \star p<0.01, * \star * p<0.001$ : significantly different. 


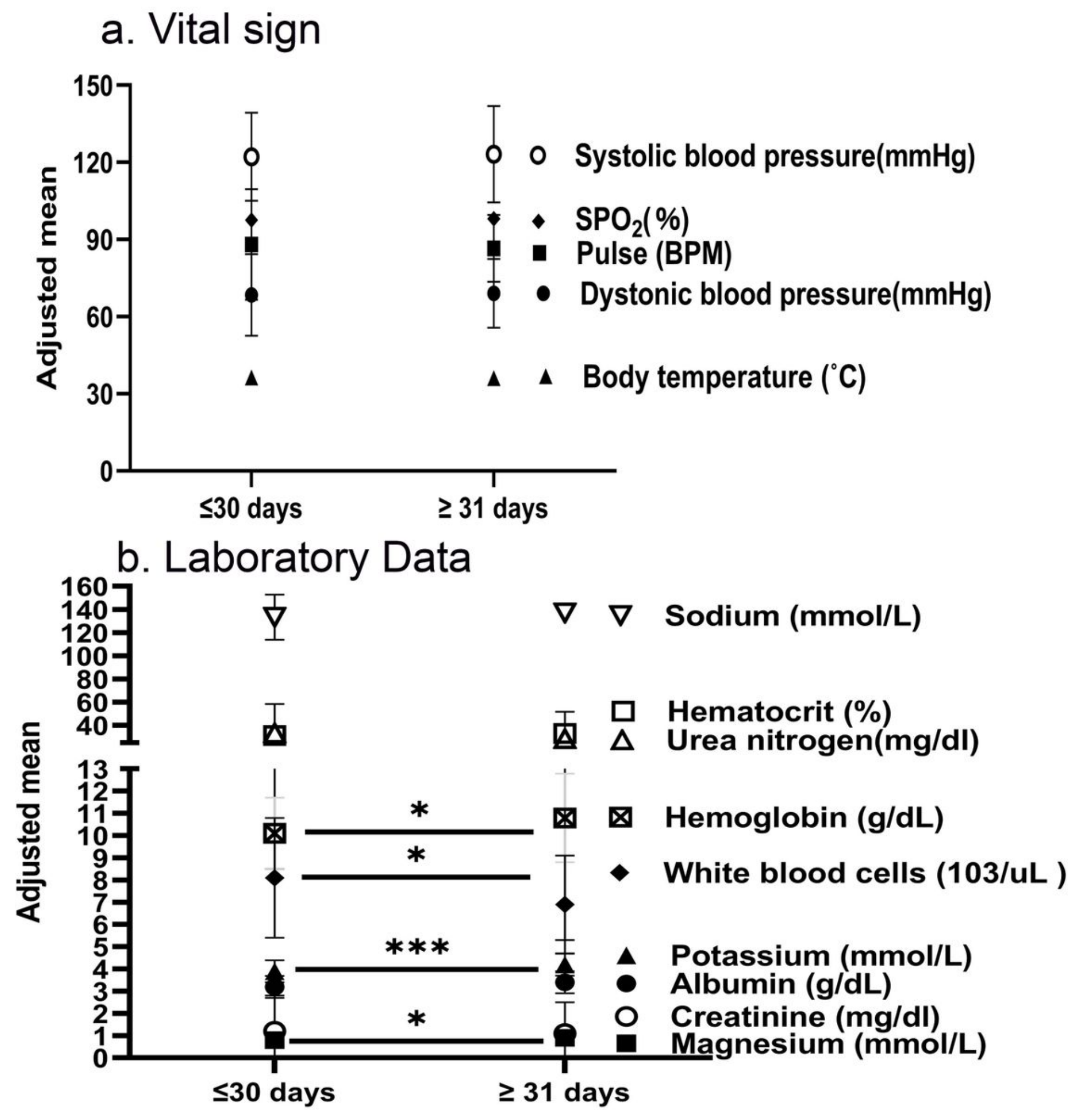

Figure 5

Comparison of early with late unplanned hospital readmission on the factors of hemodynamic and laboratory data in HMV patients. (a), (b) $₫$ mean $\pm S D$, independent $T$ test; ${ }^{*} p<0.05,{ }^{*} *{ }^{*} p<0.001$ : significantly different. 